\title{
Melatonin as a key regulator in molecular-genetic network of glucose variability related to circadian rhythm
}

\author{
Olga Saik \\ Laboratory of Computer Proteomics \\ Institute of Cytology and Genetics, \\ Siberian Branch of Russian Academy of \\ Sciences (ICG SB RAS) \\ Novosibirsk, Russia; Laboratory of \\ Endocrinology \\ Research Institute of Clinical and \\ Experimental Lymphology - Branch of the \\ Institute of Cytology and Genetics, \\ Siberian Branch of Russian Academy of \\ Sciences (RICEL - Branch of IC\&G SB \\ RAS) \\ Novosibirsk, Russia \\ saik@bionet.nsc.ru
}

\author{
Pavel Demenkov \\ Laboratory of Computer Proteomics \\ Institute of Cytology and Genetics, \\ Siberian Branch of Russian Academy of \\ Sciences (ICG SB RAS) \\ Novosibirsk, Russia \\ demps@bionet.nsc.ru \\ Vladimir Ivanisenko \\ Laboratory of Computer Proteomics \\ Institute of Cytology and Genetics, \\ Siberian Branch of Russian Academy of \\ Sciences (ICG SB RAS) \\ Novosibirsk, Russia \\ salix@bionet.nsc.ru
}

\author{
Vadim Klimontov \\ Laboratory of Endocrinology \\ Research Institute of Clinical and \\ Experimental Lymphology - Branch of the \\ Institute of Cytology and Genetics, \\ Siberian Branch of Russian Academy of \\ Sciences (RICEL - Branch of IC\&G SB \\ RAS) \\ Novosibirsk, Russia \\ klimontovvv@bionet.nsc.ru
}

\begin{abstract}
The circadian rhythm is an important factor in the diurnal fluctuations in blood glucose levels, especially in diabetes mellitus. Analysis of molecular-genetic networks performed by ANDSystem showed that melatonin can be considered as one of the key participants in associative networks that describe the relationship between circadian rhythm and glycemic variability. It also can be assumed that melatonin is able to participate in the formation of the dawn phenomenon.
\end{abstract}

Keywords - glucose variability, diabetes mellitus, moleculargenetic networks, ANDSystem, melatonin, circadian rhythm

\section{Motivation and Aim}

Pathological fluctuations in glucose levels are associated with various aspects of diabetes and especially with the development of its complications. The glycemic variability depends, among other factors, on the circadian rhythm.

The ANDSystem, developed at the Institute of Cytology and Genetics SB RAS, allows to automatically build associative molecular genetic networks associated with complex phenotypic characters and biological processes. Analysis of molecular-genetic networks can improve understanding of the mechanisms of glucose variability.

\section{Matherials and Methods}

An associative network of interactions of molecular objects with a circadian rhythm, hyperglycemia and hypoglycemia, as well as a network describing the relationships between these molecular objects, were created using the "Query Wizard" function of ANDSystem. The calculation of the betweenness centrality for network nodes was carried out by the "Statistics" function in the "Analysis" section of ANDSystem. An associative network describing the effect of melatonin on insulin and glucose via intermediary proteins was constructed using the "Pathway Wizard" function of the ANDSystem system.

\section{Results}

According to ANDSystem molecular-genetic networks, 49 objects are associated with circadian rhythm, hyperglycemia, and hypoglycemia, including hormones, growth factors, cytokines, chemical substances, and metabolites. Among the participants in the network were leptin, adiponectin, insulin, interleukin-6, melatonin, growth hormones, cortisol, dopamine, serotonin, estrogens, androgens, and others.

Analysis of the network showed that melatonin and its precursor serotonin possessed the highest values of betweenness centrality. Melatonin plays a key role in maintaining sleep-wake cycles. It is interesting that at 4-8 o'clock in the morning there is a sharp decline in the concentration of melatonin and at the same time some patients with diabetes show dawn phenomenon. Analysis of molecular-genetic networks showed that melatonin is able to regulate insulin and glucose levels. This regulation requires participation of a large number of intermediary genes and proteins.

\section{Conclusion}

Analysis of associative networks suggests that normal daily glycemic variability is controlled by a big set of biological signaling pathways that have a multidirectional effect. This ensures that the glucose concentration is maintained at a normal level. However, the loss of a number of links of this network due to mutations and/or pathological processes disrupts the system that maintain glucose balance in the physiological range during circadian oscillations.

\section{ACKNOWLEDGMENT}

The research was supported by Grant of Russian Science Foundation № 20-15-00057 «Development of personalized approaches to assessing glycemic variability in patients with type 1 diabetes based on mathematical methods and artificial intelligence». 